\title{
Risk factors for unplanned removal of central venous catheters in hospitalized children with hematological and oncological disorders
}

\author{
Moe Miyagishima $^{1}$, Hamada Motoharu ${ }^{1}$, Yuji Hirayama ${ }^{1}$, Hideki Muramatsu${ }^{1}$, Takahisa \\ Tainaka $^{1}$, Chiyoe Shirota ${ }^{1}$, Akinari Hinoki ${ }^{1}$, Takahiro Imaizumi ${ }^{2}$, Masahiro Nakatochi ${ }^{1}$, Eri \\ Nishikawa $^{1}$, Nozomu Kawashima ${ }^{1}$, Atsushi Narita ${ }^{1}$, Nobuhiro Nishio ${ }^{1}$, Seiji Kojima ${ }^{1}$, and \\ Yoshiyuki Takahashi ${ }^{1}$ \\ ${ }^{1}$ Nagoya University Graduate School of Medicine Faculty of Medicine \\ ${ }^{2}$ Nagoya University Hospital
}

November 27, 2020

\begin{abstract}
Background: Central venous catheters (CVCs) have been essential devices for the treatment of children with hematological and oncological disorders. Only few studies investigated the complications and selections of different types of CVCs in these pediatric patients. This study aimed to compare risk factors for unplanned removal of two commonly used CVCs, i.e., peripherally inserted central catheters (PICCs) and tunneled CVCs, and propose better device selection for the patient. Procedure: This retrospective, single center cohort analysis was conducted on pediatric patients with hematological and oncological disorders inserted with either a PICC or a tunneled CVC. Results: Between January 1, 2013, and December 31, 2015, 89 patients inserted with tunneled CVCs (total 21,395 catheter-days) and 84 with PICCs (total 9,177 catheter-days) were followed up until the catheter removal. The median duration of catheterization was 88 days in PICCs and 186 days in tunneled CVCs ( $\mathrm{p}=$ $1.24 \times 10-9)$. PICCs at the 3 -month cumulative incidence of catheter occlusion $(5.2 \%$ vs. $0 \%, \mathrm{p}=4.08 \times 10-3)$ and total unplanned removal $(29.0 \%$ vs $7.0 \%, \mathrm{p}=0.0316)$ were significantly higher, whereas no significant difference was observed in the cumulative incidence of central line-associated bloodstream infection ( $11.8 \%$ vs. $2.3 \%, \mathrm{p}=0.664)$. Multivariable analysis identified younger age ( $<2$ years) (subdistribution hazard ratio [SHR], 2.29; 95\% confidence interval [CI], 1.27-4.14) and PICCs (SHR, 2.73; 95\% CI, 1.48-5.02) were independent risk factors for unplanned removal. Conclusion: Our results suggest that tunnel CVCs would be a preferred device for children with hematological and oncological disorders requiring long-term, intensive treatment.
\end{abstract}

\section{Introduction}

Central venous catheters (CVC) are essential devices for a safe and reliable vascular access when treating pediatric patients with hematological or oncological diseases requiring long-term, intensive treatment. ${ }^{1} \mathrm{CV}$ Cs play a fundamental role in the administration of chemotherapeutic agents, transfusion, and parenteral nutrition as well as painless blood sampling for frequent regular examination. Conversely, catheter-related complications are always highly problematic for clinicians because they result in not only unplanned removal and catheter reinsertion but also treatment delay, increased mortality, and healthcare costs. ${ }^{2}$ Deep vein thrombosis (DVT) and central line-associated bloodstream infection (CLABSI) are two major CVC complications. The presence of immunosuppression related to disease or therapy, thrombocytopenia, and coagulopathy in patients with cancer makes it more important to prevent and manage these complications. ${ }^{3}$

Peripherally inserted central catheters (PICCs) are non-tunneled CVCs inserted through a peripheral vein of the upper arm. Owing to its ease of insertion and removal, it is more commonly used as alternative devices of other types of CVCs for pediatric and adult patients. ${ }^{4}$ PICC insertion is less invasive and more cost-effective than that of conventional type of CVCs, including tunneled CVCs or implanted ports requiring 
general anesthesia and surgery in the operating room. Even during removal, PICCs can be easily removed without sedation or local anesthesia. Although PICCs have these advantages, many studies have reported the limited longevity and higher risk of DVT of PICCs as compared with other types of CVCs in both adults ${ }^{4}$ and children. ${ }^{5}$

Therefore, the British Committee for Standards in Haematology (BCSH) guideline in adults recommended that PICCs are suited as ambulatory or outpatient-based therapy contraindicated as an inpatient therapy for adult patients with hematological disorders. Although some studies reported the acceptability of long-term use of PICCs for hospitalized children with cancer, ${ }^{6,7}$ the prospective observational study on children with various diseases requiring CVC placement showed that PICCs had significantly higher incidences of venous thromboembolism (VTE), CLABSI, and catheter malfunction than those of tunneled CVCs. This study also showed that patients with leukemia harbor higher risk of CVC-related VTE. ${ }^{8}$ However, no comparative studies have been conducted on PICCs and conventional CVCs in pediatric patients with hematological and oncological disorders.

Here, we performed a retrospective study on PICC longevity in comparison with tunneled CVCs to identify risk factors of unplanned CVC removal for appropriately selecting the CVC type in pediatric patients with hematological and malignant diseases.

\section{Material and Methods}

\section{Patients}

A total of 173 consecutive children with hematological or oncological disorders who received placement of their initial CVCs [PICC $(\mathrm{n}=84)$ or tunneled CVC $(\mathrm{n}=89)$ ] at Nagoya University Hospital from January 1, 2013, to December 31, 2015, were retrospectively reviewed. We excluded second or more CVC insertions according to this study. The clinical characteristics of patients are summarized in Table 1 . This study was approved by the ethics committee of Nagoya University Graduate School of Medicine.

\section{Catheter placement}

The attending physicians selected the types of catheter based on their patients' clinical conditions. PICCs (Groshong ${ }^{\circledR}[$ Bard Peripheral Vascular, Inc., Tempe, USA] catheter) were inserted by well-trained pediatricians under clean contaminated condition in the fluoroscopy room. Operators selected any of the major veins of the upper extremities, usually the cephalic or basilica vein. Tunneled CVCs (Hickman ${ }^{\circledR}$ [Bard Peripheral Vascular, Inc., Tempe, USA] or Broviac ${ }^{\circledR}$ [Bard Peripheral Vascular, Inc., Tempe, USA] catheters) were inserted into one of the internal jugular veins by well-trained pediatric surgeons in the operating room. The catheter tip was positioned at the superior vena cava under the radiographic guidance in both types of catheters.

\section{Unplanned catheter removal}

The causes of unplanned catheter removal were categorized into self-removal, occlusion, mechanical events (catheter malposition or fracture), CLABSI, and infection without confirmed CLABSI. Confirmed CLABSI was defined as catheter infection with positive blood culture. Catheters inserted to patients with persistent fever of unknown origin who did not respond to antibiotic therapy were empirically removed and categorized as infection without confirmed CLABSI when their blood culture was negative.

\section{Statistical analysis}

PICCs and tunneled CVCs were compared using the Mann-Whitney U test for continuous variables and Fisher's exact test for categorical variables. Correlations between patients' covariates or factors and unplanned removal were evaluated using univariate and multivariable logistic regression analyses. Cumulative incidence of unplanned catheter removal was calculated using competing risk methods. Fine-Gray proportional hazards model was used to estimate the subdistribution hazard ratios on causes of catheter removal with PICCs versus tunneled CVCs. Incidences of complications or removal were calculated per 1,000 catheter days. All $p$ -values reported are two-sided, and $p$-values of $<0.05$ were considered statistically significant. All statistical 
analyses were performed using EZR (Saitama Medical Center, Jichi Medical University). ${ }^{9}$ Proportionality was evaluated using Schoenfeld residuals, met for all analyses.

We compare cumulative incidence of unplanned removal of PICCs and tunneled CVCs, and removal at the end of therapy was regarded as censoring. The incidence for each event was calculated per 1,000 catheter days.

\section{Results}

\section{Patient characteristics}

The median age of patients with PICCs was higher than those with tunneled CVCs (6.5 vs. 2.7 years, $p$ $<0.001)$. No gender or disease predominance was noted between the two groups. Patients with PICCs were less likely to receive SCT than those with tunneled CVCs $(9.5 \%$ vs. $32.6 \%, p<0.001)$ because patients scheduled for SCT were initially inserted with tunneled CVC in our institute.

\section{The incidence of unplanned catheter removal}

Unplanned catheter removal was observed in 28 of $84(33 \%)$ patients with PICCs and 33 of 89 (37\%) with tunneled CVCs (Table 2 ). The median catheter life was 88 days with a total of 9,177 catheter days for PICCs and 186 days with a total of 21,395 catheter days for tunneled CVCs, respectively. The incidence of unplanned removal per 1,000 catheter days was 3.05 and 1.54 for PICCs and tunneled CVCs, respectively (Table 3 ). The cumulative incidence of unplanned catheter removal of PICCs was significantly higher than that of tunneled CVCs at (3-month cumulative incidence, $29.0 \% ; 95 \%$ confidence interval [CI], 19.1-39.6\% vs. $6.9 \% ; 2.8-13.5 \% ; p=0.0316)$ (Figure 1A ).

\section{Infection with or without confirmed CLABSI}

Among the causes of unplanned removal, infection without confirmed CLABSI was the most common for both types of catheter, with the incidence per 1,000 catheter days of 0.87 and 0.56 for PICCs and tunneled CVCs, respectively. The incidence of confirmed CLABSI was also similar between PICCs ( 0.33 per 1,000 catheter days) and CVC (0.42 per 1,000 catheter days). The cumulative incidence of infection was not significantly different between both types of catheters $(p=0.664)$ (Supplementary Figure 1A ).

\section{Catheter occlusion}

The incidence per 1,000 catheter days of catheter occlusion was 0.54 and 0 for PICCs and for tunneled CVCs, respectively. The cumulative incidence of catheter occlusion for PICCs was significantly higher than that for tunneled CVCs $(p=0.0041)$ (Supplementary Figure 1B ).

\section{Mechanical events, self-removal, and other reasons}

The incidence per 1,000 catheter days of mechanical events was 0.65 and 0.51 for PICCs and tunneled CVCs, respectively. The cumulative incidence of mechanical events was not significantly different between both types of catheters $(p=0.716)$ (Supplementary Figure 1C). The incidence per 1,000 catheter days of self-removal was 0.54 and 0.047 for PICCs and tunneled CVCs, respectively. The incidence of self-removal was extremely low for tunneled CVCs, as it was one of the major causes of catheter removal for PICCs. The cumulative incidence of self-removal for PICCs was significantly higher than that for tunneled CVCs $(p$ $=0.0469)$ (Supplementary Figure 1D ). The incidence per 1,000 catheter days of other reasons was 0.11 and 0 for PICCs and tunneled CVCs, respectively. One of the other reasons was discomfort at the catheter insertion site, the only case with PICC.

Univariate and multivariable analyses of risk factors for unplanned catheter removal

Univariate and multivariable analyses were performed to identify risk factors for unplanned removal of CVCs (Table 4 ). Age $<2$ years (subdistribution hazard ratio [SHR], 2.290;95\% CI, 1.26-4.16; $p=0.006$ ) and PICCs (SHR, 2.727; 95\% CI, 1.518-4.9; $p<0.001$ ) are identified as independent risk factors for unplanned 
removal, whereas no significant associations were observed among gender, disease, and stem cell transplantation. The cumulative incidence of unplanned catheter removal in patients with younger age ( $<2$ years) was significantly higher than those of other patients (3-month cumulative incidence [95\%], 21.7\% [10.6-35.3\%] vs. $15.4 \%[9.7-22.4 \%] ; p=0.0496]$ (Figure 1B ).

\section{Discussion}

Here, we performed the first retrospective study that identifies risk factors for unplanned removal of CVCs in pediatric patients with hematological and malignant diseases. In multivariable analysis, PICCs and younger age $(<2$ years $)$ are found as independent risk factors for unplanned catheter removal. Although the incidence of planned removal was similar between PICCs and tunneled CVCs (54.0\% vs. 53.6\%), the median catheter life of PICCs was significantly shorter than that of tunneled CVCs ( 88 vs. 186 days). To the best of our knowledge, no reports have assessed the effects of children's age on catheter complications. In this study, younger patients with PICCs had a particularly high frequency of unplanned removal (11 of $17,65 \%)$. Notably, 4 of 5 children who self-removed their PICCs aged $<2$ years. These results suggest that the tunneled CVC is a more appropriate device for the treatment of hematological and malignant diseases, especially in younger children.

A meta-analysis of adult patients ${ }^{4}$ and a systemic review of pediatric patients ${ }^{10}$ have reported a higher risk of VTE with PICCs as compared to tunneled CVCs. In particular, a prospective study was conducted on pediatric patients with leukemia and similarly showed that PICCs were at a higher risk of VTE ${ }^{8}$. A limitation of this study is that only a few cases with catheter occlusion did not receive radiological confirmation of VTE. However, in this cohort, catheter removal due to occlusion was significantly more common in PICCs than that in CVCs; our observations are consistent with those of previous studies, as CVC occlusion is mostly due to a thrombus. ${ }^{11}$

In this study, no difference was observed in the frequency of CLABSI between PICCs and CVCs in patients with hematological and malignant diseases, a result inconsistent with that of previous pediatric studies with the higher incidence of catheter infection in PICCs, ${ }^{8,10}$ although these studies included a wide range of pediatric diseases and not limited to those with pediatric hematological and malignant diseases. In adult patients, the frequency of CLABSIs in PICCs and CVCs has been investigated in several cohorts of patients with various diseases, with reports suggesting a higher risk for PICCs ${ }^{12}$ and other reports suggesting a higher risk for $\mathrm{CVCs}^{13}$. Meanwhile, a systematic review of adult patients with cancer concluded that the occurrence of infection did not differ between catheter types. ${ }^{14}$ A larger prospective study should be conducted to determine which catheter type has a lower risk of CLABSI, especially in pediatric patients with hematological and malignant diseases. In conclusion, this study revealed that PICCs is associated with a higher risk of unplanned catheter removal than tunneled CVCs in children with hematological or malignant diseases. Among the causes of catheter removal, the risk of CLABSI according to PICCs was comparable to tunneled CVCs; however, the risk of catheter occlusion was significantly higher than that of tunneled CVCs. Moreover, young age (aged $<2$ years) was another independent risk factor for unplanned catheter removal. These results suggest that tunneled CVCs would be a preferred device for children, especially with younger age, with hematological and oncological disorders requiring long-term and intensive treatment. Future studies are warranted to determine more reliable catheter selection methods.

\section{Conflict of Interest Statement}

The authors declare no competing financial interests.

\section{Acknowledgements}

The author gratefully acknowledge Dr. Yusuke Okuno for his advice. The authors would like to thank all clinicians and families who made this study possible by providing clinical information.

\section{References}

1. Schiffer CA, Mangu PB, Wade JC, et al. Central Venous Catheter Care for the Patient With Cancer: 
American Society of Clinical Oncology Clinical Practice Guideline. J Clin Oncol 2013;31:1357-1370.

2. Wilson MZ, Rafferty C, Deeter D, et al. Attributable costs of central line-associated bloodstream infections in a pediatric hematology/oncology population. Am J Infect Control 2014;42:1157-1160.

3. Chopra V, Kuhn L, Coffey CE, et al. Hospitalist experiences, practice, opinions, and knowledge regarding peripherally inserted central catheters: A michigan survey. J Hosp Med 2013;8:309-314.

4. Chopra V, Anand S, Hickner A, et al. Risk of venous thromboembolism associated with peripherally inserted central catheters: A systematic review and meta-analysis. Lancet 2013;382: 311-325.

5. Noailly Charny PA, Bleyzac N, Ohannessian R, et al. Increased risk of thrombosis associated with peripherally inserted central catheters compared with conventional central venous catheters in children with leukemia. J Pediatr 2018;198:46-52.

6. Hatakeyama N, Hori T, Yamamoto M, et al. An evaluation of peripherally inserted central venous catheters for children with cancer requiring long-term venous access. Int J Hematol 2011;94:372-377.

7. Abedin S, Kapoor G. Peripherally Inserted central venous catheters are a good option for prolonged venous access in children with cancer. Pediatr Blood Cancer 2008;51:251-255.

8. Jaffray J, Witmer C, O'Brien SH, et al. Peripherally inserted central catheters lead to a high risk of venous thromboembolism in children. Blood 2020;135:220-226.

9. Kanda Y. Investigation of the freely available easy-to-use software "EZR" for medical statistics. Bone Marrow Transplant 2013;48:452-458.

10. Ullman AJ, Marsh N, Mihala G, et al. Complications of central venous access devices: A systematic review. Pediatrics 2015;136:e1331-e1344.

11. Baskin JL, Pui CH, Reiss U, et al. Management of occlusion and thrombosis associated with long-term indwelling central venous catheters. Lancet 2009;374:159-169.

12. Christensen LD, Holst M, Bech LF, et al. Comparison of complications associated with peripherally inserted central catheters and Hickman ${ }^{\mathrm{TM}}$ catheters in patients with intestinal failure receiving home parenteral nutrition. Six-year follow up study. Clin Nutr 2016;35: 912-917.

13. Mollee P, Jones M, Stackelroth J, et al. Catheter-associated bloodstream infection incidence and risk factors in adults with cancer: A prospective cohort study. J Hosp Infect 2011;78:26-30.

14. Pittiruti M, Hamilton H, Biffi R, et al. ESPEN Guidelines on Parenteral Nutrition: Central Venous Catheters (access, care, diagnosis and therapy of complications). Clin Nutr 2009;28:365-377.

Table 1. Clinical characteristics

\begin{tabular}{|c|c|c|c|c|}
\hline & & PICCs & Tunneled CVCs & $P$-value \\
\hline Total, $\mathrm{n}$ & Total, n & 84 & 89 & \\
\hline Catheter life, days, median (range) & Catheter life, days, median (range) & $88(5-344)$ & $186(6-1078)$ & $<0.001$ \\
\hline Age, years, median (range) & Age, years, median (range) & $6(0-17)$ & $2(0-16)$ & $<0.001$ \\
\hline \multirow{3}{*}{ Gender, $\mathrm{n}(\%)$} & Gender, n $(\%)$ & & & \\
\hline & Male & $47(56.0)$ & $54(60.7)$ & NS \\
\hline & Female & $37(44.0)$ & $35(39.3)$ & NS \\
\hline \multirow[t]{5}{*}{ Disease, n (\%) } & Disease, n (\%) & & & \\
\hline & Hematological malignancy & $37(44.1)$ & $36(40.4)$ & NS \\
\hline & Solid tumor & $39(46.4)$ & $39(43.8)$ & NS \\
\hline & Nonmalignant hematological disorder & $5(5.9)$ & $9(10.1)$ & NS \\
\hline & PID & $3(3.6)$ & $5(5.6)$ & NS \\
\hline $\mathrm{SCT}, \mathrm{n}(\%)$ & $\mathrm{SCT}, \mathrm{n}(\%)$ & $8(9.5)$ & $29(32.6)$ & $<0.001$ \\
\hline
\end{tabular}


PICC, peripheral inserted central catheter

CVC, central venous catheter

PID, primary immunodeficiency

SCT, stem cell transplantation

NS, not significant

Table 2. Cause of catheter removal

Unplanned removal, n (\%)

Planned removal, n (\%)

Death before catheter removal, n (\%)

Under treatment, n (\%)

PICC, peripheral inserted central catheter CVC, central venous catheter CLABSI, central line-associated blood stream infe

Table 3. Incidence of catheter removal per 1000 catheter days

Causes of unplanned catheter removal

Infection with confirmed CLABSI

Infection without confirmed CLABSI

Occlusion

Mechanical cause

Self-removal

Others

Total Unplanned removal

PICC, peripheral inserted central catheter CVC, central venous catheter CLABSI, central line-associated blood stream infe

Table 4. Univariate and multivariable analysis of risk factors for unplanned removal

Related risk factors

Gender

Age

Device

Disease

SCT 
Table 4. Univariate and multivariable analysis of risk factors for unplanned removal

SHR, subdistribution hazard ratio CI, confidence interval CVC, central venous catheter PICC, peripheral inserted central c

\section{Figure Legends}

Figure 1. (A) Comparison of cumulative incidence of unplanned removal in PICCs vs. tunneled CVCs after the insertion. (B) Comparison of unplanned removal by age.

\section{Supplementary Figure Legends}

Supplementary figure 1. (A) Comparison of cumulative incidence of infection in PICCs vs. tunneled CVCs. (B) Comparison of cumulative incidence of occlusion in PICCs vs. tunneled CVCs. (C) Comparison of cumulative incidence of mechanical events in PICCs vs. tunneled CVCs. (D) Comparison of cumulative infection of self-removal in PICCs vs. tunneled CVCs. 